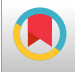

\title{
Presenting a Framework for Effective E-Mentoring Relationships in Medical Sciences: A Meta-Synthesis Method
}

\author{
Ghasem Salimi, ${ }^{1,}$ Mehdi Mohammadi, ${ }^{2}$ and Nayyereh Hosseini ${ }^{3}$ \\ ${ }^{1}$ Assistant Professor, Faculty of Education and Psychology, Shiraz University, Shiraz, Iran \\ ${ }^{2}$ Associate Professor, Faculty of Education and Psychology, Shiraz University, Shiraz, Iran \\ ${ }^{3} \mathrm{PhD}$ Student of Educational Administration, Shiraz University, Shiraz, Iran \\ "Corresponding author: Ghasem Salimi, Assistant Professor, Faculty of Education and Psychology, Shiraz University, Shiraz, Iran. Tel: +98-9177711501, E-mail: \\ salami.shu@gmail.com
}

Received 2017 June 21; Revised 2017 November 19; Accepted 2017 December 05.

\begin{abstract}
Background: Mentoring is used as a developmental model in many academic disciplines including clinical medicine and public health sciences. An effective relationship between the instructor or faculty member and the student can be particularly helpful in the professional growth and improvement of students, especially, in clinical disciplines. The current study aimed at presenting a framework for effective e-mentoring relationships in medical sciences to improve the quality of e-mentoring in this field.

Methods: The current study presented a framework for effective e-mentoring relationships using a qualitative meta-synthesis method. The previous studies on the effective factors in mentoring relationships from 2000 to 2017 were analyzed. The research population included all indexed articles in PubMed, Sage, Taylor and Francis, Science Direct, Emerald, Springer, Scopus, and Web of Science. After 2 stages of screening based on the title and abstract of the articles, 90 articles were selected and studied. Finally, 27 articles with the highest relevance to the topic were selected as the research sample. Research data were extracted from these articles by means of open coding, and then, the data were analyzed and synthesized.

Results: Twenty-eight minor components were identified for effective e-mentoring in medical sciences in higher education. The main e-mentoring framework was organized in terms of 4 major components; i e, communication medium, quantity of communication, quality of communication, and communication outcome.

Conclusion: In light of the findings of previous studies on mentoring in higher education over 17 years, the current study presented a framework for policy-making, evaluation, and development of mentoring relationship between instructors and students in clinical medical sciences in higher education.
\end{abstract}

Keywords: Electronic Mentoring, Online Mentoring, Education, Medical, Meta-Synthesis Method

\section{Background}

Mentoring includes a wide range of activities such as personal advising, improving professional skills and knowledge, promoting self-confidence, and facilitating professional development. Mentoring is done by an instructor (mentor) to a less experienced learner (mentee) (1). Different kinds of mentoring in various contexts and disciplines are already studied, including instructorstudent (or faculty-student) mentoring in the context of higher education (2). Mentoring is used as a developmental model in many academic disciplines including clinical and public health sciences $(2,3)$. Griffiths and Miller believed that mentoring is especially important for the students of medical sciences and is in the center of contemporary discussions in education. E-mentoring is recently recognized as a new field of mentoring by the researchers (4). Traditionally, mentoring refers to a mutually beneficial relationship and a face-to-face interpersonal experience between the mentor and mentee (5). However, time, geographical distance, professional and occupational complexities make face-to-face interaction between the mentor and mentee difficult, and thus, create certain challenges in designing mentoring programs $(1,6)$. Online mentoring emerged as a solution to these challenges (1). According to Ensher and Murphy, e-mentoring is defined as a "mutually beneficial relationship between a mentor and a protégé" in which "electronic means" are used to support new learning, offer career guidance, and provide emotional support (7). The electronic platform in e-mentoring creates a flexible and asynchronous communication between the mentor and mentee, regardless of their time and place $(1,2,5)$. E-mentoring can facilitate both synchronous (same-time) and asynchronous or delayed communication. Asynchronous mentoring relationship can 
give the mentor more time to respond the questions and provide more accurate responses in comparison with immediate responses in a face-to-face situation $(1,4,5)$. Participating in an asynchronous mentoring relationship also involves a form of commitment to work-life ethic (8). In e-mentoring, the mentors and mentees can communicate with each other via different means including e-mail, discussion boards, instant messaging, videoconferencing via web cameras, listservs, Usenet, newsgroups, threaded discussions and/or chat rooms, telephone, typed chat, asynchronous message board, Second Life, Facebook, Twitter, Skype, and texting $(4,5,9,10)$. Schichtel contends that ementoring makes it possible to have mentors who are geographically at a distant from medical interns, and therefore, it provides professional expertise and assistance for those who do not have access to a face-to-face interaction (1).

Although the conceptual framework of face-to-face is different from that of e-mentoring, i e, they are different in terms of their temporal and spatial qualities of communication (1); they are deeply similar in their ultimate goals (2). In other words, face-to-face mentoring and ementoring share many functions such as acculturation, promoting self-confidence, and effective modeling (1). Accordingly, studies showed that e-mentoring is able to improve the advantages and qualities of face-to-face mentoring, and therefore, guarantee the educational, academic, social, professional, and personal success of the mentees (6). Griffiths and Miller assert that e-mentoring has an important role in the professional and occupational development of interns (4). According to Schichtel, e-mentoring can improve the quality of education in medical sciences and work as a supplementary method for face-to-face mentoring in bringing about the continuous professional and occupational growth and improvement of the mentees (1). The results of different studies showed that e-mentoring can foster inference-making in clinical contexts and enable the mentees to implement their knowledge, boost their confidence, and make better decisions (11). According to Stewart, e-mentees in obstetrics made great use of ementoring with regard to improving their clinical performance, acquiring new information, solving problems, and tackling ethical dilemmas in their profession (12).

According to the above discussion, an effective ementoring relationship between the instructor and the student, especially in clinical disciplines, is highly important. Wong and Prekumar contend that the combination of methods of face-to-face mentoring with those of e-mentoring is necessary to achieve any success in ementoring (2). An effective and satisfactory e-mentoring should be student-centered, flexible, frequent, and academically and psychosocially supportive (10). According to Doyle et al., the 3 important dimensions of e-mentoring, which play a role in its success are: a plan or structure for mentoring communication, the frequency of mentoring interactions, and the opportunity for mentoring participants to meet face-to-face (10). Frequent coordinated interactions and the use of different methods of communication such as webcams, emails, and mobile phones are among some of the most important characteristics of ementoring from the perspective of Jacobs et al. Studies also suggest that the efficiency and success of e-mentoring increases when the duration of the e-mentoring period increases (5). Briones and Janoske discussed the significance of the type of communication medium that best serves the needs of the mentor and mentee. The findings of their study indicate that online relationships are not sufficient and should be joined and complemented by faceto-face interaction (13). Based on the results of a study by Bierema and Merriam, a successful e-mentoring relationship depends on mutual respect, trust, and a convivial atmosphere of relationship (14). Moreover, the success of ementoring depends on commitment to the relationship and exchange of constructive feedback (15).

Most researchers believe that while the implementation of e-mentoring is on the rise, there is not still a fully developed concept of e-mentoring, which encompasses its various dimensions and parameters (2). Given the significance and advantages of effective E-mentoring relationships, the current research aimed at delineating and presenting a practical and comprehensive framework for ementoring. Accordingly, the main research question can be phrased as follows: what are the factors in effective ementoring relationships? The minor research questions are:

1- What are the major factors in effective e-mentoring relationship?

2- What are the minor factors in effective e-mentoring relationship?

\section{Methods}

The current study used a meta-synthesis method. Metasynthesis is a qualitative meta-analysis of concepts and results of the previous studies by means of common coding method in qualitative researches (16). In other words, meta-analysis includes a thorough reevaluation of previous qualitative analyses and their findings in a particular area (17) and the attempt to combine the data collected from this evaluation into a new framework to achieve a comprehensive knowledge about a topic $(16,18)$. Since studies centering on e-mentoring are mostly qualitative case studies, meta-synthesis can be used as an effective 
method to achieve the goal of the current article, i e, presenting a comprehensive framework for e-mentoring relationships. In order to implement the abovementioned method, the 7-step method presented by Sandelowski M, Barroso (19) was used according to Figure 1. Further explanations for each step are presented.

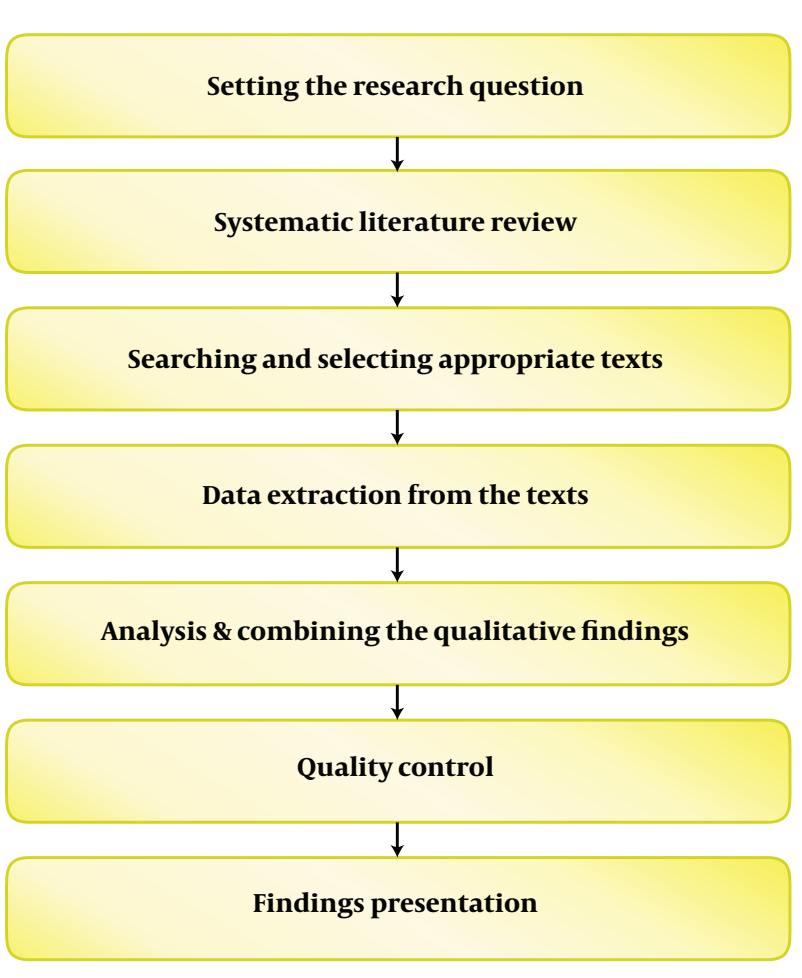

Figure 1. Meta-Synthesis Steps in the Research

\subsection{Step 1. Setting the Research Question}

In the 1st step of implementing the meta-synthesis method, the main research question should be identified. Therefore, in the introduction section of the paper the research question was mentioned.

\subsection{Steps 2 and 3. Systematic Literature Review and Searching the Appropriate Texts}

The of the current study included all English language articles indexed in PubMed, Sage, Taylor and Francis, Science Direct, Emerald, Springer, Scopus, and Web of Science from 2000 to 2017 in which one of the key words listed in Box 1 were used either in the title or their abstract. After 2 stages of screening based on the titles and abstracts, 90 articles were extracted and studied. Finally, 27 articles with the highest relevance to the purpose of the current study were selected as the research sample and analyzed.
Box 1. The Searching Key Words

\begin{tabular}{l}
\hline Key Words \\
\hline E-mentoring \\
\hline Electronic mentoring \\
\hline Online mentoring \\
\hline Virtual mentoring \\
\hline E-mentorship \\
\hline Electronic Mentorship \\
\hline Online mentorship \\
\hline Virtual mentorship \\
\hline
\end{tabular}

It should be noted that to measure the quality of the selected literature at this stage, the critical appraisal skill program critical appraisal skills program (CASP) was used (20).

\subsection{Step 4. Data Extraction from the Texts}

In the current meta-synthesis, the text of the research (research and review texts) was considered as data and documented exactly as the interview text (16). At this stage, using the open coding of the selected articles, a large number of codes were identified and extracted based on the components of effective e-mentoring relationships in the form of a 3-column table (including main factors, codes, and works cited).

\subsection{Step 5. Analysis and Combining the Qualitative Findings}

At this stage, the codes obtained from the previous step were analyzed and compared. Further, some of the codes were summarized and simplified, some of the codes closely related to the content were combined and synthesized and the similar codes were deleted. Then, the remaining codes were re-labeled and categorized several times. Finally, the derived categories were identified as the main components of the e-mentoring relationships.

\subsection{Step 6. Quality Control}

Regarding the quality control in the current study, it should be noted that all 27 selected papers, as a research sample, were published in journals with high-impact factor (IF) and peer-reviewed ones. Finally, 27 papers were selected out of 90 peer-reviewed articles and papers were scored based on the 10 CASP criteria and 50-option Rubik scale (Table 1). Papers scored below good level were excluded (21).

With the help of 10 questions, the CASP helped the researchers to determine the accuracy and importance of the selected studies for review. These questions focused on 
Table 1. Rubik 50 Points Scale

\begin{tabular}{lccc}
\hline Score Range & Abbreviation & Definition & Procedure \\
\hline $\mathbf{4 0 - 5 0}$ & E & Excellent & Accepted \\
$\mathbf{3 1 - 4 0}$ & VG & Very good & Accepted \\
$\mathbf{2 1 - 3 0}$ & G & Good & Rejected \\
$\mathbf{1 1 - 2 0}$ & F & Fair & Rejected \\
\hline $\mathbf{0 - 1 0}$ & P & Poor & Rejected \\
\hline
\end{tabular}

the following themes: research objectives, methodology, study design, sampling method, data collection strategy, reflectivity (including the relationship between researcher and participants), ethical considerations, data analysis accuracy, clear expression of the findings, and the value of the research (in terms of relevance and coherence with the research subject) (20).

Likewise, in terms of content analysis, the repetition of the codes and components extracted from various articles indicated the importance and confirmation of the extracted components based on the viewpoint of researchers.

\subsection{Step 7. Findings Presentation}

At this stage, the results of the previous steps were presented.

\section{Results}

The analysis of the selected articles on effective ementoring relationships resulted in 28 minor factors. The extracted codes were recoded and in the end, the main factors were categorized into 4 groups. Accordingly, the results of the current study, shown in Table 2, suggested the framework of effective e-mentoring relationships including 4 main factors, namely communication medium, quantity of communication, quality of communication, and communication outcome, as well as 28 minor factors.

\section{Discussion}

Various research findings show that successful and effective e-mentoring relationships between the mentor and mentee can be greatly helpful to improve the professional and academic development of the university students. The current study aimed at filling this g ap b y p resenting a comprehensive framework for the effective factors in ementoring. The framework for effective e-mentoring relationships presented in the current study included 4 main factors; i e, medium of communication, quantity of communication, quality of communication, and communication outcome. The factors in the medium of communication were: 1. Using different synchronous and asynchronous communication media such as email, telephone, social networks, video conference, live chat, SMS, etc., 2. The working skills of the mentor and mentee including, computer skills, familiarity with electronic communication media, internet skills, and writing skills, 3 . The agreement between the mentor and mentee on the type of communication media, their accessibility, and convenience of use, and 4. Combining face-to-face interaction with ementoring.

Stewart and Carpenter argued that using different communication media such as email and social networks can create more flexibility in interaction and learning. The findings of their study showed that technology along with effective use of communication skills can overcome the obstacle of physical and spatial distance, and therefore, become an important supportive tool in clinical contexts (11). According to the results of a study conducted on the effects of e-mentoring on the learning of students in and obstetrics and gynecology in New Zealand, telephone, fax, email, video conference, and live chat were the most commonly used communication methods by the mentors and mentees (22). According to Todd, the use of smart phones by university students to stay connected to their mentor is increasing. Smart phones enable the mentees to stay connected to social networks. These networks, e g, Facebook, can strengthen the mentor-mentee relationship because of their informal platform of communication, frequent interactions, and immediate sharing of the sources. Researchers also emphasized video chatting and twitting as the advantages of using smart phones in e-mentoring. SMS can be used effectively to improve e-mentoring relationships (9). What is important in choosing a medium of communication is the convenience and accessibility of the medium both for the mentor and mentee (8). Moreover, the agreement of the mentor and mentee on the type of communication medium is important $(2,11,22)$.

Ensher et al., asserted that people with better computer and writing skills most likely have a better experience of e-mentoring than the ones without skills (23). Given the fact that the ability of the mentee to express his/her thoughts and feelings through written messages is highly important in e-mentoring relationship, creating a suitable online platform for this exchange is of great importance. Familiarity with electronic media and internet skills can immensely improve the quality of e-mentoring relationships. Researchers believe that familiarity with an online communication channel, e g, email or live chat; can help the mentor and mentee to send and receive useful in- 
Table 2. The Suggested Framework for Effective E-mentoring Relationships

\begin{tabular}{|c|c|c|}
\hline Main Factors & Minor Factors & Works Cited \\
\hline \multirow{4}{*}{ 1. Communication medium } & $\begin{array}{l}\text { 1.1. Using different synchronous and asynchronous communication media such as } \\
\text { email, telephone, social networks, video conference, live chat, SMS, etc. }\end{array}$ & $(2,5,9,10,22)$ \\
\hline & $\begin{array}{l}\text { 1.2. The working skills of the mentor and mentee, including computer skills, } \\
\text { familiarity with electronic communication media, internet skills, and writing skills }\end{array}$ & $(8,23,24)$ \\
\hline & $\begin{array}{l}\text { 1.3. The agreement between the mentor and mentee on the type of communication } \\
\text { media, their accessibility, and convenience of use }\end{array}$ & $(2,8,10,11,13,22,25)$ \\
\hline & 1.4. Combining face-to-face interaction with e-mentoring & $(2,9,10,13,22,24,26-28)$ \\
\hline \multirow{2}{*}{ 2. Quantity of communication } & $\begin{array}{l}\text { 2.1. Frequency of interaction and number of communications between the mentor } \\
\text { and mentee (constant interaction between the mentor and mentee) }\end{array}$ & $(5,6,9-11,15,24,27,29,30)$ \\
\hline & $\begin{array}{l}\text { 2.2. The duration of the relationship and the continuation of the mentor-mentee } \\
\text { relationship }\end{array}$ & $(2,5,24)$ \\
\hline \multirow{12}{*}{ 3. Quality of communication } & $\begin{array}{l}\text { 3.1. Mutual communication between the mentor and mentee based on active } \\
\text { cooperation and participation, and a sense of shared responsibility for the success of } \\
\text { the relationship }\end{array}$ & $(5,10,11,13,15,27,30)$ \\
\hline & $\begin{array}{l}\text { 3.2. Flexibility and the agreement between the mentor and mentee about the } \\
\text { programs and scheduling the interactions and mentoring sessions }\end{array}$ & $(10,11)$ \\
\hline & $\begin{array}{l}\text { 3.3. The similarities between the mentor and mentee in terms of personal traits, } \\
\text { values, attitudes, working styles, personal and professional skills, field of expertise, } \\
\text { and interests }\end{array}$ & $(2,6,11,15,24,27,30)$ \\
\hline & $\begin{array}{l}\text { 3.4. Commitment and mutual trust between the mentor and mentee, empathy, and } \\
\text { self-revelation }\end{array}$ & $(2,11,14,25,30)$ \\
\hline & $\begin{array}{l}\text { 3.5. Honesty and open-mindedness of the mentor, and sharing knowledge } \\
\text { information, and experiences }\end{array}$ & $(2,11,13-15)$ \\
\hline & 3.6. Interest and enthusiasm of the mentor and mentee in the mentoring relationship & $(15,29,31)$ \\
\hline & 3.7. Preplanned mentoring sessions and interactions on a frequent basis & $(2,10,22,29)$ \\
\hline & $\begin{array}{l}\text { 3.8. Satisfaction of the mentor and mentee with the mentoring relationship, and } \\
\text { enjoying this experience }\end{array}$ & $(9,10,13,15,30,32)$ \\
\hline & $\begin{array}{l}\text { 3.10. Friendly, informal, and strong relationship between the mentor and mentee, and } \\
\text { the freedom to discuss personal issues }\end{array}$ & $(13-15,24,30)$ \\
\hline & 3.11. Satisfaction and the freedom of the choice of the mentee in choosing the mentor & $(13,30)$ \\
\hline & $\begin{array}{l}\text { 3.12. The sufficiency of time in each interaction and during each communication } \\
\text { session }\end{array}$ & $(5,10,11)$ \\
\hline & 3.13. Giving immediate feedbacks by the mentor to the mentee & $(13,15,28)$ \\
\hline \multirow{9}{*}{ 4. Communication outcome } & 4.1. The professional goals of the mentee & $(5,10,11)$ \\
\hline & 4.2. Professional publications & $(5,10)$ \\
\hline & $\begin{array}{l}\text { 4.3. Giving assistance in the process of thesis writing, and helping the mentee during } \\
\text { that process }\end{array}$ & $(5,10)$ \\
\hline & 4.4. Academic, psychosocial, and social support of the mentee by the mentor & $(4,5,7,10,25,29,32,33)$ \\
\hline & 4.5. Improving the research skills of the mentee & $(5,33)$ \\
\hline & $\begin{array}{l}\text { 4.6. Improving the knowledge, expertise, and professional and occupational skills of } \\
\text { the mentee }\end{array}$ & $(2,3,5,11,32,33)$ \\
\hline & 4.7. Personal growth and development of the mentee & $(2,9,30,33)$ \\
\hline & 4.8. Role modeling & $(1,14,32)$ \\
\hline & 4.9. Professional and occupational counsel for the mentee & $(4,7,33)$ \\
\hline
\end{tabular}


more successful in expressing their thoughts and feelings via electronic communication and were also more skilled in interpreting and responding to the information (29).

The 2nd main factor presented in the current article was the quantity of communication. The minor factors of quantity of communication included: 1 , frequency of interaction and number of communications between the mentor and mentee (constant interaction between the mentor and mentee); 2 , the sufficiency of time in each interaction and during each communication session; and 3, the duration of the relationship and the continuation of the mentor-mentee relationship.

In general, the process of human communication is in direct correlation with the frequency of communication. In the field of e-mentoring, this is referred to the frequency of communication (frequency of contact) between the mentor and mentee. Most researchers believe that frequent and regular interaction between the mentor and mentee can promote mutual trust and understanding and lead to many positive results in the e-mentoring relationship. Frequent communication plays a pivotal role in e-mentoring relationships. In a qualitative study, Bonnett et al., found out that the mentees considered higher frequency of communication as a measure of successful e-mentoring relationship (29). Similar to the findings of DiRenzo et al., Eby et al., Grant-Vallone and Ensher, and Jacobs et al., also suggested that the frequency of communication (face-to-face and electronic) was effective on the success of e-mentoring relationships (5). According to the results of a study on the e-mentoring relationship between the instructor and the student in the online postprofessional doctor of occupational therapy (OTD) program, it was proven that at least one 28 -60-minute session of communication per week is highly necessary (10).

It is highly important for the mentee to discuss the issues with the mentor during a mentoring session in order to get the needed information and solve the problems (11). One of the biggest challenges of the mentors is to spend enough time on mentoring relationships. Occupational duties and responsibilities leave little time for mentoring, and therefore, it may have a negative impact on the mentoring relationship (13). However, it should be noted that maintaining effective and long-term mentoring relationship is required to spend enough time and both the mentors and mentees should communicate. According to Doyle et al., the duration of each communication can affect the mentoring relationships (10).

Mentoring relationships can be cultivated in the course of time. A well-established relationship over a long time is one of the important factors in a successful ementoring relationship (10). In another study, showed that the efficiency and success of e-mentoring relationships in- crease when the mentoring period lasts for a longer time (5).

Quality of communication was one of the other main factors presented in the current article. The minor factors in the quality of communication included: 1 , mutual communication between the mentor and mentee based on active cooperation and participation, and a sense of shared responsibility for the success of the relationship; 2 , flexibility and the agreement between the mentor and mentee about the programs and scheduling of the interactions and mentoring sessions; 3, the similarities between the mentor and mentee in terms of personal traits, values, attitudes, working styles, personal and professional skills, field of expertise, and interests; 4, commitment and mutual trust between the mentor and mentee, empathy, and self-revelation; 5, honesty and open-mindedness of the mentor, and sharing knowledge, information, and experiences; 6 , interest and enthusiasm of the mentor and mentee in the mentoring relationship; 7, preplanned mentoring sessions and interactions on a frequent basis; 8, satisfaction of the mentor and mentee with the mentoring relationship, and enjoying this experience; 9 , the recognition of the mentor and mentee about the success of the mentoring experience, and the fulfillment of the expectations and hopes of both of them; 10, informal and strong relationship between the mentor and mentee, and the freedom to discuss personal issues; 11 , satisfaction and the freedom of the choice of the mentee to choose the mentor; and 12 , immediate feedbacks from the mentor on mentee.

According to a study conducted by Stewart and Carpenter, the main factor in the success of e-mentoring programs was interaction based on cooperation and participation, and shared sense of commitment between the mentor and mentee (11). Rhodes et al., considered the mutual responsibility and accountability as 2 factors in the quality of e-mentoring relationship (15). Similarly, Wallis et al., argued that a successful e-mentoring relationship depended on the participation and mutual efforts of the mentor and mentee to improve the relationships (27). A look at the existing literature on successful e-mentoring relationship suggested that similarities between the mentor and mentee were an important factor. According to Stewart and Carpenter, the mentees considered similarities between themselves and the mentors in terms of attitude and commitment to shared learning as an important factor (11). The findings of Doyle et al., suggested that similar demographic features and similarities in terms of values, attitudes, and personal traits of the mentor and mentee, while not necessary, were highly important (10). In any case, even when there was little similarity, it was necessary for the mentors to have enough knowledge about the personal traits, needs, and fields of the interests of the mentees, and 
to make the required changes in order to meet the needs and expectations of the mentees.

Trust is clearly of an especial importance in improving the quality of e-mentoring relationships (30). Most researchers believe that information confidentiality and privacy between the mentor and mentee are important factors in a successful mentoring relationship, and by analogy, in an efficient e-mentoring relationship (2).

The emotional involvement of the mentor and mentee in the mentoring relationships is one of the criteria in the quality of relationship (15). According to the findings of DiRenzo et al., and Wong and Looi, the motivation and enthusiasm of the mentor and mentee to actively participate in the e-mentoring relationship were important factors in the success of the e-mentoring relationship $(29,31)$. Motivation and the positive attitudes of the mentors and mentees can help to overcome some of these challenges without affecting the mentoring relationship. According to the findings of a research, pre-planned communication sessions between the mentor and mentee can be more useful than immediate interactions without a prior coordination (2, 10). Loureiro-Koechlin and Allan found that planned communication and regular structured interactions can help the mentees make a better use of the e-mentoring relationship (5).

Many studies showed that the feeling of satisfaction by the mentor and mentee about interacting with each other and enjoying the mentoring experience were important factors in a successful e-mentoring relationship $(9,10,15)$.

The success of the e-mentoring relationship depends on the perception of the mentor and mentee about the fulfillment of their goals and expectations, and the advantages of the experience of mentoring $(5,10)$. The findings of a study suggested that the communication patterns in successful and unsuccessful e-mentoring relationships were different. Whereas the unsuccessful pairs used a more formal style and distant tone, the successful pairs used an informal and supportive style (24).

Most researchers contend that the dissatisfaction of the mentee with the process of choosing a mentor and the feeling of hopelessness in that process has a negative impact on the mentoring programs (30). The selection of the mentor by the mentee can help to create a successful ementoring relationship (13). Rhodes et al., argued that responding to the messages is one of the important factors to achieve a successful e-mentoring relationship. For them, not responding to a message in due time was similar to missing a session in a face-to-face mentoring (15). The 4th main factor in the framework presented by the article was the communication outcome, which included the following minor factors: 1 , the professional goals of the mentee; 2 , professional publications; 3 , giving assistance in the pro- cess of the writing and helping the mentee during that process; 4, academic, psychosocial, and social support of the mentee by the mentor; 5 , improving the research skills of the mentee; 6 , improving the knowledge and expertise, as well as professional and occupational skills of the mentee; 7 , personal growth and development of the mentee; 8 , role modeling; and 9, professional and occupational counsel for the mentee.

The above minor factors were used in different studies to evaluate e-mentoring programs $(5,9,10,27,30,33)$. The sum of these factors can be termed the communication outcome, or the content of interaction between the mentor and mentee.

The framework presented in the current article can be used as a basis to evaluate and develop e-mentoring relationships in the education of medical sciences students. Improving effective e-mentoring relationships between the mentor and mentee requires a consideration of the factors discussed in the current article.

\section{Footnote}

\section{Conflict of Interest: None Declared.}

\section{References}

1. Schichtel M. Core-competence skills in e-mentoring for medical educators: a conceptual exploration. Med Teach. 2010;32(7):e248-62. doi: 10.3109/0142159X.2010.489126. [PubMed: 20653366].

2. Williams S, Sunderman J. E-mentoring in an online course: Benefits and challenges to e-mentors. Int J Evid Based Coach Mentor. 2012;10(1):109-22.

3. Harris R, Birk SB, Sherman J. E-Mentoring for Doctor of Nursing Practice Students: A Pilot Program. J Nurs Educ. 2016;55(8):458-62. doi: 10.3928/01484834-20160715-07. [PubMed: 27459433].

4. Griffiths M, Miller H. E-mentoring: does it have a place in medicine?. Postgrad Med J. 2005;81(956):389-90. doi: 10.1136/pgmj.2004.029702. [PubMed: 15937205].

5. Jacobs K, Doyle N, Ryan C. The nature, perception, and impact of e-mentoring on post-professional occupational therapy doctoral students. Occup Ther Health Care. 2015;29(2):201-13. doi: 10.3109/07380577.2015.1006752. [PubMed: 25821883].

6. de Janasz SC, Godshalk VM. The Role of E-Mentoring in Protégés' learning and satisfaction. Group Organ Manage. 2013;38(6):743-74. doi: 10.1177/1059601113511296.

7. Ensher EA, Murphy SE. E-mentoring: Next generation research and strategies. In: B.R. Ragins amp;K, editor. The hand book of mentoring at work: Theory, research and practice. Thousand Oaks, CA: Sage; 2007. p. 272-301.

8. Pietsch TM. A transition to e-mentoring: factors that influence nurse engagement. Comput Inform Nurs. 2012;30(12):632-9. doi: 10.1097/NXN.0b013e318266cbc5. [PubMed: 22918137].

9. Todd RL. Social media as online mentoring tools for STEM students with and without disabilities. International Conference on Universal Access in Human-Computer Interaction. Springer Berlin Heidelberg; 2013. p. 256-65. 
10. Doyle N, Jacobs K, Ryan C. Faculty Mentors' Perspectives on EMentoring Post-Professional Occupational Therapy Doctoral Students. Occup Ther Int. 2016;23(4):305-17. doi: 10.1002/oti.1431. [PubMed: 27250596].

11. Stewart S, Carpenter C. Electronic mentoring: An innovative approach to providing clinical support. International Journal of Therapy and Rehabilitation. 2009;16(4):199-206. doi: 10.12968/ijtr.2009.16.4.41191.

12. Stewart S. A pilot study of email in an e-mentoring relationship. $J$ Telemed Telecare. 2006;12(S3):83-5. doi:10.1258/135763306779380011.

13. Briones Rowena L, Melissa J. Mentoring 2.0: How PR educators use social media to create and maintain relationships with students. Int J Contin Eng Educ Life Long Learn. 2013;23(1):17-30. doi: 10.1504/ijceell.2013.051764.

14. Bierema LL, Merriam SB. E-mentoring: Using computer mediated communication to enhance the mentoring process. Innov High Educ. 2002;24(3):201-17.

15. Rhodes JE, Spencer R, Saito RN, Sipe CL. Online mentoring: the promise and challenges of an emerging approach to youth development. J Prim Prev. 2006;27(5):497-513. doi: 10.1007/s10935-006-0051-y. [PubMed: 16897405].

16. Sohrabi Yurtchi B, Aazami A, Yazdani HR. The pathology of the research of Islamic management based on meta-analysis [In Persian]. Journal Public Adm Perspect. 2011;6:9-24.

17. Bench S, Day T. The user experience of critical care discharge: a metasynthesis of qualitative research. Int J Nurs Stud. 2010;47(4):487-99. doi: 10.1016/j.ijnurstu.2009.11.013. [PubMed: 20004396].

18. Zimmer L. Qualitative meta-synthesis: a question of dialoguing with texts. J Adv Nurs. 2006;53(3):311-8. doi: 10.1111/j.1365-2648.2006.03721.x. [PubMed: 16441536].

19. Sandelowski M, Barroso J. Handbook for synthesizing qualitative research. Springer Publishing Company; 2006.

20. Mohammadian A, Manian A, Khodadad Bormi M. A systematic review and future orientation of research in the field of business virtual incubators. QJ Inform Technol Manage. 2015;12:123-50.

21. Karanjam SS, Zarif Sanaiey N, Karanjam S. Designing a comprehensive organizational e-learning model using the meta-synthesis method. Interdiscip J Virtual Learn Med Sci. 2017;8(3). doi: 10.5812/ijvlms.11496.
22. Stewart S, Wootton R. A survey of e-mentoring among New Zealand midwives. J Telemed Telecare. 2005;11 Suppl 2:S90-2. doi: 10.1258/135763305775124731. [PubMed: 16375810].

23. Ensher EA, Heun C, Blanchard A. Online mentoring and computermediated communication: New directions in research.J Vocat Behav. 2003;63(2):264-88. doi: 10.1016/s0001-8791(03)00044-7.

24. Shpigelman CN, Gill CJ. The characteristics of unsuccessful ementoring relationships for youth with disabilities. Qual Health Res. 2013;23(4):463-75. doi: 10.1177/1049732312469115. [PubMed: 23221100].

25. Shpigelman C. Electronic Mentoring and Media. In: David L DB Karcher MJ, editors. Handbook of Youth Mentoring. Thousand Oaks: SAGE Publications, Inc; 2014. p. 239-52.

26. Valentin-Welch M. Evaluation of a National E-Mentoring Program for Ethnically Diverse Student Nurse-Midwives and Student Midwives. J Midwifery Womens Health. 2016;61(6):759-67. doi: 10.1111/jmwh.12547. [PubMed: 27926807].

27. Wallis JAM, Riddell JK, Smith C, Silvertown J, Pepler DJ. Investigating patterns of participation and conversation content in an online mentoring program for Northern Canadian Youth. Mentor Tutoring Partnership Learn. 2015;23(3):228-47. doi: 10.1080/13611267.2015.1072395.

28. Schichtel M. A conceptual description of potential scenarios of ementoring in GP specialist training. Educ Prim Care. 2009;20(5):360-4. doi: 10.1080/14739879.2009.11493818. [PubMed: 19849902].

29. DiRenzo MS, Linnehan F, Shao P, Rosenberg WL. A moderated mediation model of e-mentoring. J Vocat Behav. 2010;76(2):292-305. doi: 10.1016/j.jvb.2009.10.003.

30. Simmonds D, Zammit Lupi AM. The matching process in e-mentoring: a case study in luxury hotels. J Eur Ind Train. 2010;34(4):300-16. doi:10.1108/03090591011039063.

31. Wong LH, Looi CK. Online discussion and e-mentoring strategies in blended continuing education courses. Comparative blended learning practices and environments. 2010. p. 146-69.

32. de Janasz SC, Ensher EA, Heun C. Virtual relationships and real benefits: using e-mentoring to connect business students with practicing managers. Mentor Tutoring Partnership Learn. 2008;16(4):394-411. doi: $10.1080 / 13611260802433775$.

33. Macafee DAL. E-mentoring in Surgical Training. Health Serv Insights 2012;5:9-28. doi: 10.4137/hsi.s9613. 